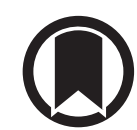

CrossMark

\title{
The importance of interventional timing in the bleomycin model of pulmonary fibrosis
}

\author{
Philipp Kolb ${ }^{1,2}$, Chandak Upagupta ${ }^{1,2}$, Megan Vierhout ${ }^{1}$, Ehab Ayaub ${ }^{1}$, \\ Pierre Simon Bellaye ${ }^{1}$, Jack Gauldie ${ }^{1}$, Chiko Shimbori ${ }^{1}$, Mark Inman ${ }^{1}$, \\ Kjetil Ask ${ }^{1}$ and Martin R.J. Kolb (ib ${ }^{1}$
}

Affiliations: ${ }^{1}$ Firestone Institute for Respiratory Health, Depts of Medicine, McMaster University, Hamilton, ON, Canada. ${ }^{2}$ These authors contributed equally to this work.

Correspondence: Martin R.J. Kolb, Division of Respirology, Firestone Institute for Respiratory Health, McMaster University, 50 Charlton Avenue East, Room T2120, Hamilton, ON L8N 4A6 Canada. E-mail: kolbma mcmaster.ca

@ERSpublications

Preclinical models are important to decipher mechanisms of disease, identify novel treatment targets and develop new drugs. The bleomycin model is the standard model for pulmonary fibrosis, but it is often used incorrectly, as shown by this meta-analysis. http://bit.ly/39qboxP

Cite this article as: Kolb P, Upagupta C, Vierhout M, et al. The importance of interventional timing in the bleomycin model of pulmonary fibrosis. Eur Respir J 2020; 55: 1901105 [https://doi.org/10.1183/ 13993003.01105-2019].

ABSTRACT Idiopathic pulmonary fibrosis (IPF) is a complex disease of unknown aetiology, which makes drug development challenging. Single administration of bleomycin directly to the lungs of mice is a widely used experimental model for studying pulmonary fibrogenesis and evaluating the effect of therapeutic antifibrotic strategies. The model works by inducing an early inflammatory phase, which transitions into fibrosis after 5-7 days. This initial inflammation makes therapeutic timing crucial. To accurately assess antifibrotic efficacy, the intervention should inhibit fibrosis without impacting early inflammation.

Studies published between 2008 and 2019 using the bleomycin model to investigate pulmonary fibrosis were retrieved from PubMed, and study characteristics were analysed. Intervention-based studies were classified as either preventative (starting $<7$ days after bleomycin installation) or therapeutic ( $>7$ days). In addition, studies were cross-referenced with current major clinical trials to assess the availability of preclinical rationale.

A total of 976 publications were evaluated. 726 investigated potential therapies, of which $443(61.0 \%)$ were solely preventative, $166(22.9 \%)$ were solely therapeutic and $105(14.5 \%)$ were both. Of the 443 preventative studies, only $70(15.8 \%)$ characterised inflammation during the model's early inflammatory phase. In the reported 145 IPF clinical trials investigating 93 compounds/combinations, only 25 (26.9\%) interventions had any preclinical data on bleomycin available on PubMed.

Since 2008 , we observed a shift (from $<5 \%$ to $37.4 \%$ ) in the number of studies evaluating drugs in the therapeutic setting in the bleomycin model. While this shift is encouraging, further characterisation of early inflammation and appropriate preclinical therapeutic testing are still needed. This will facilitate fruitful drug development in IPF, and more therapeutic strategies for patients with this devastating disease.

This article has an editorial commentary: https://doi.org/10.1183/13993003.00811-2020

This article has supplementary material available from erj.ersjournals.com

Received: 5 June 2019 | Accepted after revision: 24 Feb 2020

Copyright OERS 2020 


\section{Introduction}

Idiopathic pulmonary fibrosis (IPF) is a devastating lung disease in which abnormal wound healing causes a scarring of the pulmonary interstitium and distortion of the lung architecture [1]. This causes impaired gas exchange as well as increased lung stiffness, which eventually leads to respiratory failure. The prognosis of IPF is poor and comparable to aggressive cancers, with a median mortality of 3-4 years post-diagnosis [2]. The epidemiology of IPF has been studied in different regions and countries around the world. Canadian data suggest an incidence rate of 18.7 and a prevalence of 41.8 per 100000 individuals [3]. In populations aged $>70$ years, the prevalence can be as high as 200 cases per 100000 individuals [4]. While recently two antifibrotic medications have been approved for clinical use in treating IPF, neither treatment is curative and efficacy varies across patients. In order to improve patient outcomes and enhance long-term quality of life, additional therapies are needed [5].

In the life sciences, animals are used to understand the normal biology of health and disease, and to develop therapeutic interventions [6]. Animal models are valuable, provided that experiments are carefully designed, analysed and reported. Inadequate experimental design and reporting can result in uninterpretable and unreproducible studies, and can lead to failures in preclinical drug development [7].

Over the past few decades, several animal models have been used to study the biology of IPF [8]. These models serve as tools to test and understand biological the mechanisms of fibrogenesis and to test the efficacy of therapeutic interventions. Due to the idiopathic nature of IPF and the highly complex underlying pathobiology, no single model is able to provide a completely robust analysis. Instead, results must be considered across various preclinical platforms. Most IPF animal models involve injury to the lung, which activates wound healing pathways and eventually excessive scar formation. Common methods to induce fibrosis include systemic or intratracheal instillation of bleomycin, radiation, intratracheal administration of silica or asbestos, and transgenic mice or gene transfer employing fibrogenic mediators, among others. In addition to the commonly used method of intratracheal instillation of bleomycin, there are multiple other possible ways to administer this agent, including intraperitoneal, subcutaneous and intravenous routes, and through osmotic pumps. All of these methods are sufficient to induce lung injury and subsequent repair.

Of these approaches, intratracheal instillation of bleomycin is the most widely used and best studied [9]. It is a relatively inexpensive system, which induces a robust fibrotic response that displays several histological hallmarks seen in IPF patients $[10,11]$. These include aberrant fibroblast proliferation and differentiation, excessive deposition of extracellular matrix proteins, and destruction of the alveolar architecture. Histological staining and morphometric analysis of lung tissue, quantification of hydroxyproline and measurements of lung function are typical metrics to quantify fibrosis in animals. Overall, the bleomycin model has proven invaluable in understanding many of the cellular and molecular pathways in fibrogenesis that are central to the current understanding of IPF pathogenesis. Despite this, the bleomycin model does have limitations. One important limitation is a profound initial inflammatory phase, characterised by upregulation of acute inflammatory cytokines such as interleukin-1 and -6 and tumour necrosis factor- $\alpha$, together with an influx of neutrophils $[12,13]$. This inflammation lasts for $\sim 7$ days before subsiding into a fibrotic phase which more appropriately mimics the manifestation of IPF in human patients (figure 1) [14]. Spontaneous reversal of fibrosis, particularly in mice, typically occurs 3-4 weeks post-intratracheal bleomycin [15-18], although several groups have reported nonresolving fibrosis by using multiple intratracheal instillations of bleomycin $[19,20]$. It is important to note that in the bleomycin model there is overlap between the inflammatory and fibrotic phases, with the initial strong inflammatory response taking up to 10 days post-injury to completely clear. In a 2008 review article by our group [21], the 7-day time point is used as a separation between the inflammatory and fibrotic phases. Since there is no clear distinction between the two phases, using the 7-day time point to differentiate between preventative and therapeutic studies is a limitation that needs to be considered. It is important to include the phase of inflammation and injury, in addition to the fibrotic phase, in investigational studies, as this phase is required to initiate fibrogenesis. Therefore, it is crucial to consider these stages concertedly.

These two key features of bleomycin must be evaluated critically when using it to model IPF and assess the efficacy of therapeutic compounds. The fibrotic phase in the bleomycin model is self-limiting and eventually reversible, but it mimics important elements of the progressive fibrogenesis seen in IPF patients. This fibrotic phase is typically no longer than 2 weeks, starting 1 week after the initial injury when the acute inflammation subsides, and ending after 3 weeks when the resolution phase of fibrosis begins [14]. Therefore, the "window" during which fibrogenic mechanisms can be studied and antifibrotic drugs should be tested is relatively short. When experimental methodologies fail to take this limitation of the bleomycin model into account, the results can be misleading. For example, studies may claim that an intervention has antifibrotic effects, when in reality it may only interfere with the initial inflammation. Our group highlighted this issue in 2008, in an article that described how the vast majority of studies published between 1980 and 2006 using the bleomycin model to test antifibrotic efficacy of drugs did not 


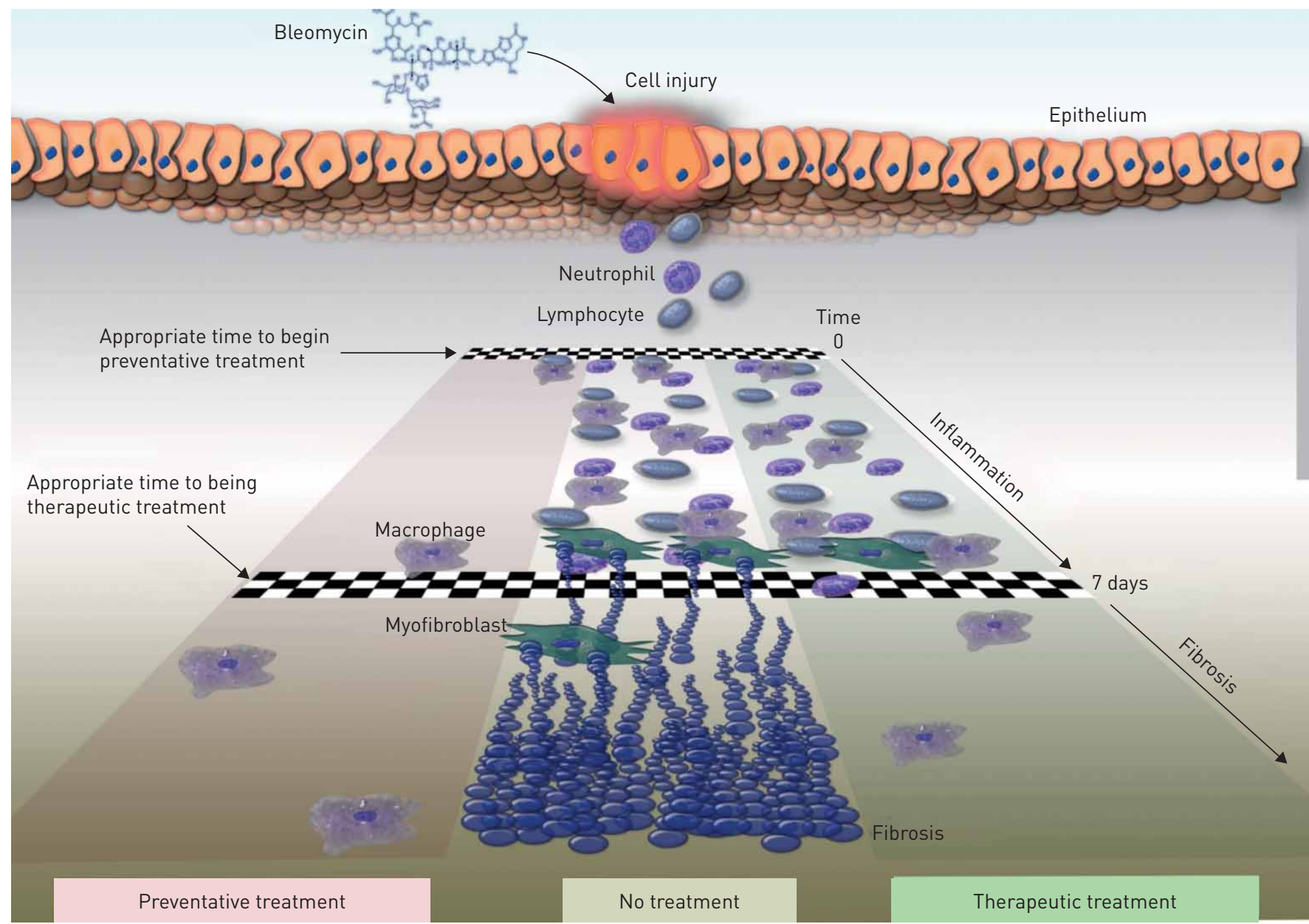

FIGURE 1 Illustration of the appropriate times to begin intervention within the bleomycin model of pulmonary fibrosis. The upper (earlier) chequered line represents the time of bleomycin exposure and the beginning of a week-long period dominated by inflammation. The second chequered line represents the beginning of a 2-week period dominated by fibrotic events. The central panel, with no treatment, features both the inflammatory and fibrotic aspects of the model. With preventative treatment (applied at the time of bleomycin exposure, left), fibrosis is absent as a result of prevention of inflammation. With therapeutic treatment (applied after the resolution of inflammation, right), prevention of fibrosis can be attributed to events independent of inflammation.

consider the element of appropriate therapeutic timing [21]. Out of 221 studies published during that period, only 10 were designed using a therapeutic treatment regime: starting the administration of an experimental therapy $>7$ days after bleomycin instillation. This inappropriate use of the experimental fibrosis models may have played a role in the slow pace of drug development in the context of IPF.

We performed a systematic review to examine whether the use of the bleomycin model to study antifibrotic effects of compounds has changed since our initial publication in 2008. By advocating for more effective use of this preclinical model, we hope to improve drug development in IPF.

\section{Methods}

A systematic PubMed search was conducted to capture the relevant literature. The search terms "bleomycin AND pulmonary fibrosis" were used to compile a list of publications between January 1, 2008 and March 10, 2019. This search yielded 1816 articles to investigate. Articles were included if they used an in vivo bleomycin model to study the effect of a particular intervention or transgenic animal on fibrosis. Excluded studies were method papers, review articles, ex vivo studies or studies aiming to understand changes in gene and protein expression in bleomycin-treated animals without intervention or genetic modifications. Non-English publications were not considered. After applying these inclusion and exclusion criteria, 976 articles were explored in detail (figure 2 and supplementary table S1).

Number of publications per year was determined by downloading the comma-separated values files from PubMed (figure 3a). All studies using mice in pulmonary fibrosis were identified by the search term "mice and pulmonary fibrosis". All bleomycin studies in pulmonary fibrosis were identified by the search term 
FIGURE 2 Flow chart outlining the inclusion/exclusion criteria for this

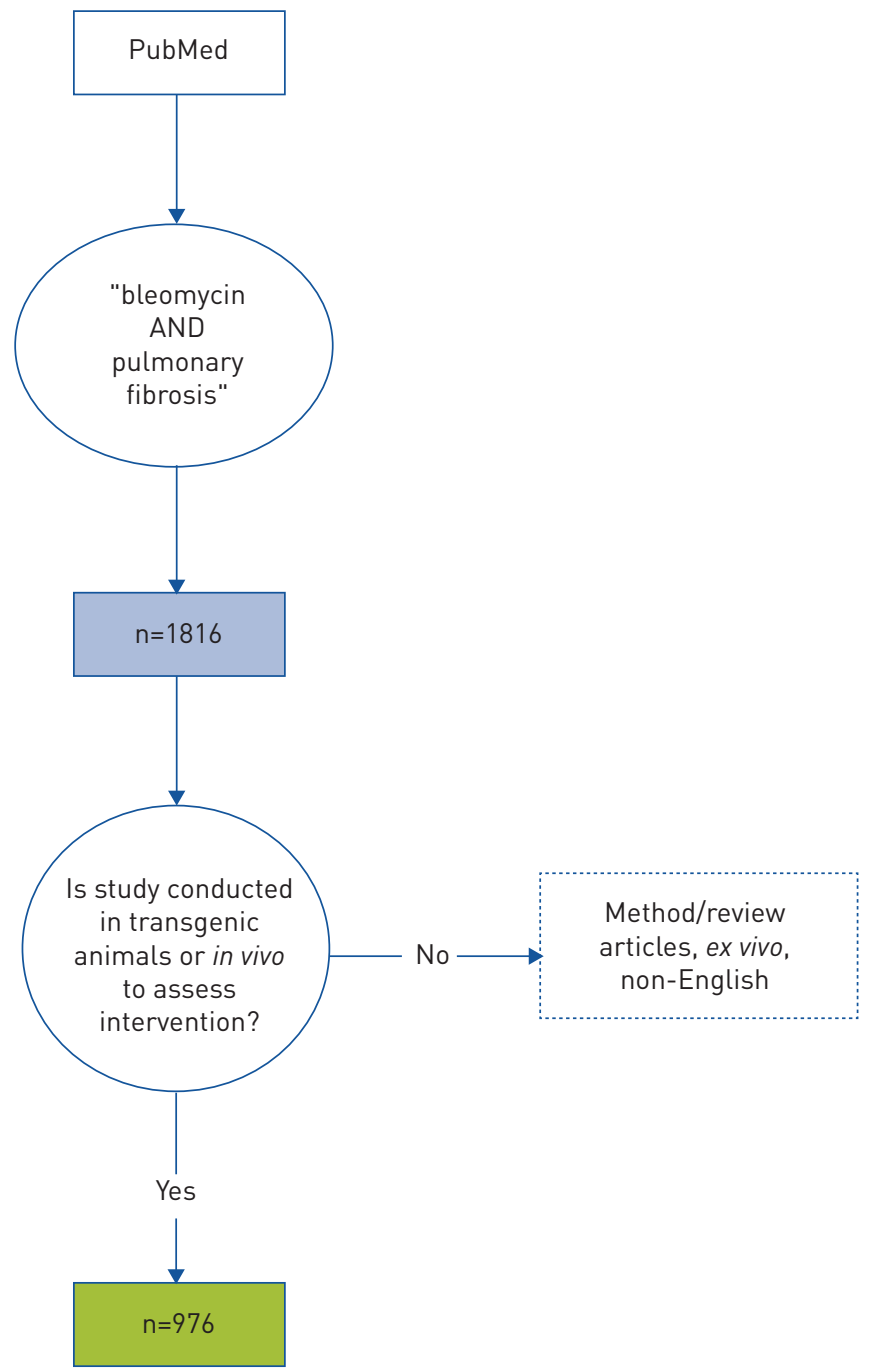
study.

"bleomycin and pulmonary fibrosis", and publications per year were plotted before and after the aforementioned inclusion and exclusion criteria.

We analysed systematically these papers and pertinent information was extracted. First, the studies were defined by study type: each paper was described as either exploring fibrogenesis in the context of an intervention, a transgenic animal or both. In studies using an intervention, the timing of intervention was characterised with respect to bleomycin administration, as outlined in MoELLER et al. [21]. Interventions beginning $<7$ days post-bleomycin were termed preventative. while interventions beginning $\geqslant 7$ days post-bleomycin were classified as therapeutic.

To understand disease prevention and progression adequately, preventative and transgenic animal studies need to assess inflammatory pathways during the early inflammatory phase in the bleomycin model. Therefore, the characterisation of inflammation $<7$ days post-bleomycin was evaluated. Typically, this involved an analysis of bronchoalveolar lavage fluid through differential cell counts, or an examination of the inflammatory cytokine profile by ELISA or gene expression analysis.

In addition, clinical trials conducted in patients suffering from IPF were searched to allow comparisons with the available basic science data from preclinical bleomycin trials. Clinical trial data were obtained from clinicaltrials.gov and the definition of associated publicly available data was defined as searchable and retrievable information available on PubMed.

\section{Results}

Overall study characterisation

Of the 976 studies published since 2008 that used the bleomycin model of pulmonary fibrosis, $74.4 \%$ $(n=726)$ investigated the potential of an intervention on fibrogenesis (i.e. small molecule, antibodies, 

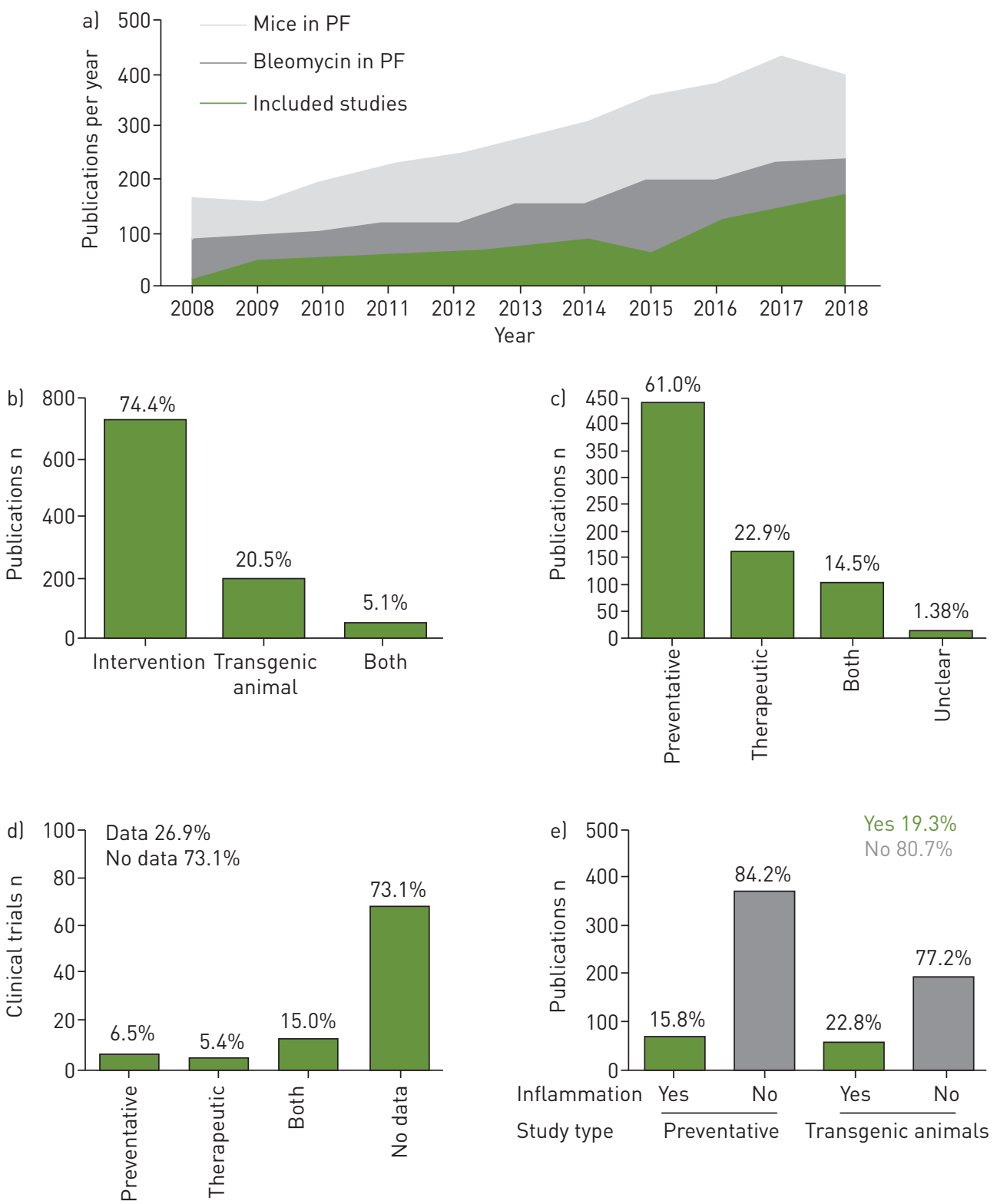

FIGURE 3 al Number of publications per year on mice in pulmonary fibrosis, and bleomycin in pulmonary fibrosis studies before and after exclusion criteria; b) distribution of studies investigated; c) distribution of intervention studies; d) associated PubMed data for idiopathic pulmonary fibrosis clinical trials; e) distribution of inflammation characterisation < 7 days after bleomycin administration in preventative and transgenic studies.

traditional medicine, etc.) (figure 3b). 20.5\% ( $\mathrm{n}=200)$ of the studies focused on the effect of bleomycin in transgenic animals, investigating whether certain gene manipulations would increase or decrease susceptibility to fibrosis. The remaining $5.1 \%(n=50)$ investigated transgenic animals or the combination of transgenic animals and interventions.

\section{Intervention characterisation}

Of the 726 intervention studies investigated, $61.0 \%$ (443 publications) were classified as preventative, while $22.9 \%$ (166 publications) were therapeutic, with intervention begun not earlier than 7 days after bleomycin administration (figure 3c). 14.5\% (105 publications) of the studies investigated both the preventative and therapeutic ability, comprising a complete assessment of intervention efficacy. 
TABLE 1 Major clinical trials assessed by AHLUWALIA et al. [22], with accompanying preclinical bleomycin data

\section{Mechanisms of action Outcome Clinicaltrials.gov Bleomycin results} ID

\begin{tabular}{|c|c|c|c|c|c|}
\hline Ambrisentan & Endothelin receptor antagonist & Negative & NCT00768300 & Not published & $\begin{array}{l}\text { December 2008- } \\
\text { February } 2011\end{array}$ \\
\hline BIBF 1120 & $\begin{array}{l}\text { Inhibitor of VEGF, PDGF, FGF } \\
\text { receptor kinases }\end{array}$ & Positive & NCT00514683 & $\begin{array}{l}\text { Preventative and } \\
\text { therapeutic }\end{array}$ & $\begin{array}{l}\text { August 2007- } \\
\text { June } 2010\end{array}$ \\
\hline Bosentan & Endothelin receptor antagonist & Negative & NCT00391443 & $\begin{array}{l}\text { Preventative and } \\
\text { therapeutic }\end{array}$ & $\begin{array}{l}\text { May } 2010 \\
\text { February } 2007- \\
\text { July } 2010\end{array}$ \\
\hline Etanercept & TNF- $\alpha$ receptor antagonist & Negative & NCT00063869 & Not published & July 2003-March 2005 \\
\hline \multirow[t]{4}{*}{ FG-3019 } & \multirow[t]{4}{*}{ Antibody against CTGF } & Results & NCT00074698 & \multirow[t]{4}{*}{ Preventative } & December 2003- \\
\hline & & awaited & NCT01262001 & & May 2004 \\
\hline & & $\begin{array}{l}\text { Positive } \\
\text { Phase }\end{array}$ & NCT01890265 & & March 2011- \\
\hline & & Results & & & June 2013- \\
\hline Imatinib & Tyrosine kinase inhibitor & Negative & NCT00131274 & $\begin{array}{l}\text { Preventative and } \\
\text { therapeutic }\end{array}$ & $\begin{array}{l}\text { April 2003- } \\
\text { August } 2007\end{array}$ \\
\hline \multirow[t]{2}{*}{ Interferon- $\gamma$} & \multirow[t]{2}{*}{ Anti-inflammatory } & Negative & NCT00047645 & \multirow[t]{2}{*}{ Not published } & April 2000- \\
\hline & & Negative & NCT00075998 & & $\begin{array}{c}\text { December } 2002 \\
\text { December } 2003- \\
\text { May } 2007\end{array}$ \\
\hline Macitentan & Endothelin receptor antagonist & Negative & NCT00903331 & Not published & $\begin{array}{c}\text { May 2009- } \\
\text { August } 2011\end{array}$ \\
\hline NAC & Antioxidant & Negative & NCT00650091 & Preventative & $\begin{array}{l}\text { October } 2009- \\
\text { January } 2014\end{array}$ \\
\hline \multirow{2}{*}{$\begin{array}{l}\text { NAC, azathioprine, } \\
\text { prednisone }\end{array}$} & \multirow[t]{2}{*}{ Antioxidant } & Positive & NCT00639496 & \multirow[t]{2}{*}{ Not published } & March 2000-July 2003 \\
\hline & & Negative & NCT00650091 & & $\begin{array}{l}\text { October } 2009- \\
\text { January } 2014\end{array}$ \\
\hline Warfarin & Anticoagulants & Negative & NCT00957242 & Not published & $\begin{array}{l}\text { October 2009- } \\
\text { July } 2011\end{array}$ \\
\hline
\end{tabular}

VEGF: vascular endothelial growth factor; PDGF: platelet-derived growth factor; FGF: fibroblast growth factor; TNF: tumour necrosis factor; CTGF: connective tissue growth factor; TGF: transforming growth factor; LOLX: lysyl oxidase-like; IL: interleukin.

Bleomycin results and the relationship with clinical trials

When evaluating the human clinical trials for IPF, it is interesting to note that in the 145 trials investigating 93 compounds/combinations, only 25 (26.9\%) interventions had any available preclinical bleomycin data on PubMed (figure 3d and supplementary table S2). Of these 25 interventions, 19 had explored drug efficacy in therapeutic models. The remaining 68 (73.1\%) compounds had no supporting data available. These results are mirrored in the major late-phase clinical trials for IPF therapies outlined by Ahluwalia et al. [22]. Out of 16 major trials, only two have provided the standard of evidence to become approved clinical therapies for IPF: nintedanib and pirfenidone. Interestingly, these two therapies, along with imatinib and bosentan, are the only interventions tested in major clinical trials for IPF that have bleomycin model data published on PubMed (table 1). A limitation of this table is that reagents utilised in murine models are sometimes different to the ones utilised in clinical trials, especially with biologic therapies, where subtle changes in protein structure require species-specific reagents. 
Inflammation characterisation

$15.8 \%(\mathrm{n}=70)$ of the 443 preventative studies investigated assessed inflammation (i.e. inflammatory cytokine profile or cell counts) prior to day 7 following bleomycin administration (figure 3e). A similar proportion was seen in the transgenic animal studies, where $22.8 \%(n=57)$ of the 250 publications investigated assessed inflammation in the inflammatory phase (at or before day 7) in the bleomycin model.

\section{Discussion}

The administration of bleomycin into the airways of small rodents is the most commonly used experimental system to study IPF pathology and serves as a widely used preclinical model to test potential therapeutic compounds [9]. In order to get the best possible information from experimental models, it is imperative to apply appropriate research methodology that reflects what is seen clinically in IPF patients.

In this study, we used the same definitions for preventative and therapeutic interventions as in our previous work and evaluated how experimental practice has changed in the past decade [21]. In contrast to the extremely low $5 \%$ of studies that were timed therapeutically prior to 2007 , in the past decade $\sim 37.4 \%$ (271 publications) of intervention studies in the bleomycin model adequately assessed therapeutic efficacy (figure 3c). This marked shift in how the bleomycin model is being used in work published between 2008 and 2019 compared to the two decades prior to 2007 highlights how researchers are receptive to change and are now using the bleomycin model to study IPF more effectively.

The use of animals for medical research has become an important point of discussion from an ethical and political perspective [23]. The recent European Respiratory Society ERS task force statement [6] addressed this important issue. It concluded that substantial efforts have been undertaken by the scientific community, including the development and application of the $3 \mathrm{R}$ principles (refinement, reduction, replacement) introduced 60 years ago to establish the highest standards for humane experimentation on animals [24]. The refined use of the bleomycin model of pulmonary fibrosis as described here reflects how scientists are willing to learn and apply the best experimental practice to comply with the $3 \mathrm{R}$ principles and animal research ethics. Further success in this context can be achieved by continuing to promote this message.

One of the key questions to experimental scientists is whether their models are useful to predict the success or failure of novel drugs in clinical studies. In theory, preclinical models do exactly this, but in reality this is not easily quantifiable. With regard to pulmonary fibrosis, the past decade has been filled with debates regarding the most appropriate end-point for both clinical trials and experimental studies. From the preclinical perspective, it is crucial to use models as appropriately as possible, and interpret the findings within the limitations of the modelling. Obviously, whether data from the bleomycin model is a prerequisite for the progression of a drug candidate into the clinical trial landscape is driven by the institution or company that develops the product. However, it is important for everyone in the field to understand how potential antifibrotic therapies work in the biological system. In our analysis we asked how novel compounds considered for the treatment of IPF were used in the bleomycin model and how their preclinical "success" compared to their clinical "success". A review by AHLuwalia et al. [22] summarised how recent clinical trials relate to the pathogenesis of IPF and was used as a baseline for our analysis. Ahluwalia et al. highlighted 16 compounds that were tested in clinical phase II and III trials in the past decade. Of these molecules, two have been approved for clinical use in IPF (nintedanib and pirfenidone).

Pirfenidone has by now been approved for the treatment of IPF in most countries [25-27]. The exact mode of action of this small molecule remains elusive; however, evidence from several clinical trials has shown convincingly that this drug reduces forced vital capacity (FVC) decline in patients with progressive IPF [26]. Preclinical data for pirfenidone in the bleomycin model is available in both preventative and therapeutic settings, which show that this molecule reduces transforming growth factor (TGF)- $\beta$ levels, fibrocyte migration and the accumulation of profibrotic myofibroblasts [28-30]. In addition, pirfenidone is able to reduce the accumulation of hydroxyproline and levels of procollagen I and III in these models [31].

Nintedanib is also approved in many countries and demonstrates a similar potential for slowing FVC decline in patients with IPF $[32,33]$. Nintedanib is a potent intracellular inhibitor of the tyrosine kinase receptors platelet-derived growth factor receptor, fibroblast growth factor receptor and vascular endothelial growth factor receptor as well as nonreceptor tyrosine kinases from the Src family [34, 35]. This small molecule attenuates processes that are essential for fibrosis such as TGF- $\beta$-induced deposition of collagen and histological fibrosis in the bleomycin model. The literature provides many examples of nintedanib's efficacy in both preventative and therapeutically designed bleomycin animal trials.

The success of these drugs in the therapeutically timed bleomycin models followed by clinical trials and US Food and Drug Administration approval suggests that there are overlaps in the underlying mechanisms 
of fibrosis in IPF and in the mouse model of bleomycin exposure. Therefore, while we may not have great insight into the mechanisms of IPF or bleomycin-induced fibrosis, this overlap provides considerable impetus for continued efforts to find new efficacious therapeutic applications in the bleomycin model.

Sildenafil is another drug that showed efficacy in both therapeutic and preventative versions of the bleomycin model. Although the STEP-IPF trial [36] which tested sildenafil in patients with advanced IPF was labelled as a "negative" trial, sildenafil was recently tested in combination with nintedanib in the INSTAGE trial in patients with advanced IPF [37]. While this trial did not meet its primary end-point of changing quality of life, it nevertheless showed that patients treated with the combination of nintedanib plus sildenafil had a reduced decline in FVC in comparison with patients treated with nintedanib alone, suggesting additive antifibrotic effects of the combination in this patient population. Another similar trial testing the combination of pirfenidone plus sildenafil for severe IPF is ongoing (NCT02951429).

Of the 16 recent phase II and III clinical trials outlined by AHLUwALIA et al. [22], six have available data on PubMed regarding their efficacy in the bleomycin model (table 1). Of the 16 trials, only those on pirfenidone, nintedanib and bosentan had available therapeutic timing results. These data provide support for the notion that potential therapies have a better chance at clinical trial success if they are effective in a therapeutic model of bleomycin. For example, and in contrast to both pirfenidone and nintedanib, the small-molecule dual endothelin receptor inhibitor bosentan was unsuccessful in two separate phase III clinical trials [38, 39]. In this case, most of the publicly available data for this molecule were preventative, and a therapeutic study was published in 2017 [40-42]. The preventative studies showed an overall decrease in collagen proteins and mRNA, as well as a reduction of $\alpha$-smooth muscle actin positive cells in the lung tissue. In addition, several studies have revealed that preventative bosentan treatment attenuates total and differential inflammatory cell counts $[41,43]$. Therefore, bosentan may interfere with the initial bleomycin-induced inflammation, which subsequently prevents profibrotic pathways from being activated. Furthermore, the recent therapeutic study noted that bosentan was effective at inhibiting bleomycin-induced collagen fibres and lung fibrosis only when administered in the preventative phase and not the therapeutic phase [42]. Together, these findings illustrate the importance of proper experimental timing in the bleomycin model.

Of course, this conclusion is based on interpretation of publicly available data alone, and it may well be that unpublished data on therapeutic bleomycin studies exist. This issue of unavailable data can be further illustrated by assessing the preclinical literature available for the 145 clinical trials that have tested or are currently testing 93 unique compounds or combinations to treat IPF. Out of these 93 interventions, no associated animal data were found for 68 on public databases (figure $3 \mathrm{~d}$ and supplementary table S2). Of the 25 interventions with available preclinical data, 20 were assessed therapeutically. Without comprehensive information available to the scientific community, it is impossible to ascertain why certain compounds may have failed in clinical trials and whether this can be attributed to inappropriate use of preclinical models. Better transparency and publication of both positive and negative experimental data have been proposed recently by an ERS task force [6].

The importance of therapeutic timing in the bleomycin model for drug development in IPF is obvious, but this does not discard the value of interventions during the initial inflammatory phase. Analysing the impact of interventions during the initiation of fibrosis allows for investigation into fibrotic pathways that may be linked to certain profibrotic aspects of inflammation. This type of understanding is particularly important when considering profibrotic macrophages, fibrocytes and other bone marrow-derived cells which are not typical of the classical acute inflammatory pathways, and contribute to fibrosis in a major way. For these preventative interventional studies, it is key that inflammation in the early phase is characterised thoroughly by cell differentials and quantification of inflammatory cytokines. It is also clear that preventative treatment regimens may impact both the inflammatory landscape and the level of cellular damage, affecting both inflammation and lung fibrosis. We found that of 443 preventative studies, only 70 (15.8\%) provided such information (figure 3e). Many of these studies reported a decrease in global inflammation following the intervention, but still claimed to show antifibrotic effects. Similar data are seen in studies using transgenic animals, where therapeutic timing is often not a viable option except for inducible genetic knockouts. Between 2008 and 2019, of the 250 reported bleomycin studies in transgenic animals, only 57 (22.8\%) characterised inflammation prior to day 7 post-bleomycin administration (figure 3e). The remaining $77.2 \%$ of studies did not investigate, or at least did not report any inflammation details. This information is critical to understand how a particular gene or pathway may contribute to fibrogenesis and potentially serve as novel therapeutic targets. Therefore, the scientific community needs to more rigorously assess these basic features in the early inflammatory phase to capitalise on its utility in preclinical experimental research.

While the bleomycin model has been widely employed, it is also important to consider its limitations and the various other models that exist and their suitability for the evaluation of the target and therapy being investigated. Subjective to the target of interest and/or method of therapeutic intervention, it is critical to 
select a suitable system for testing from the toolbox of animal pulmonary fibrosis models. The expression of the selected mechanism of action may vary between models, and awareness of this prior to investigation, as well as the critical time points of expression of these mechanisms in the model, is of substantial worth. Depending on the pathobiological processes studied, clear characterisation of the kinetics of the process studied and appropriate timing of intervention is key. A clinically relevant model is where a molecular or cellular process have been shown to be present in the pathological human setting and characterised in the corresponding animal model. This is applicable to all models of disease. In addition, this model is not without limitations. In the bleomycin model, the injury phase precedes the fibrogenic phase (although it must be remembered that there is no clear-cut distinction in timing between these phases) and treatments administered early, which reduce the injury/inflammation aspects of the model, will also impact the fibrotic part of the model. A reduction in injury will also result in a reduced need for repair mechanisms. Therefore, treatment may not have a real antifibrotic effect, but rather "anti-bleomycin-induced injury" effects. In order to claim that treatment prevents extracellular matrix deposition, it needs to be tested in a system and at a time when extracellular matrix deposition is occurring. Additionally, concerns also exist about translatability to the patient setting. In patients, although drug-induced lung fibrosis, such as by bleomycin, has been described as reversible if the drug is discontinued, as explained on the Drug-Induced Respiratory Disease Website (www.pneumotox.com), sometimes reversal does not occur. Although resolving IPF has not yet been described, there is growing belief in the research community that resolution and cure are potentially attainable, through the reprogramming of phagocytic cells.

In conclusion, the bleomycin model of lung fibrosis is still, by far the most common and fastest growing experimental tool in this field (figure 3a). In 2018, bleomycin studies comprised $60.6 \%$ of all publications that used mice to investigate pulmonary fibrosis, and the number of publications per year using bleomycin to study pulmonary fibrosis has more than doubled since 2008. Bleomycin is widely used for preclinical mechanistic research, and for preclinical studies to test drug efficacy. The basic science methodology of timing drug administration in the bleomycin model has seen a significant shift over the past 11 years compared to the preceding period. Both currently approved IPF drugs, pirfenidone and nintedanib have convincing therapeutic efficacy in the bleomycin model. For the majority of compounds that were clinically tested in the past 15 years for the treatment of IPF, only $26.9 \%$ had published bleomycin-tested data. While a more thoughtful use of the model throughout the literature is evident, there is still a substantial lack of data sharing in this field. However, we do acknowledge that utilising other search engines in addition to PubMed could potentially allow for the identification of additional studies not included in this analysis. Despite improvements over the past decade, scientists from both private and academic sectors should strive to optimise this experimental model of lung fibrosis, as it will prove imperative to facilitate cutting-edge drug development for IPF.

Conflict of interest: P. Kolb has nothing to disclose. C. Upagupta has nothing to disclose. M. Vierhout has nothing to disclose. E. Ayaub has nothing to disclose. P.S. Bellaye has nothing to disclose. J. Gauldie has nothing to disclose. C. Shimbori has nothing to disclose. M. Inman has nothing to disclose. K. Ask reports grants from Boehringer Ingelheim, GSK, Prometic, Alkermes, Pharmaxis, Respivert, Indalo, Canadian Institutes for Health Research, Kniksa, Avalyn, National Sciences and Engineering Research Council, Ontario Thoracic Society, Canadian Pulmonary Fibrosis Foundation, Actelion, Gilead, Patara, Synairgen and Unity, outside the submitted work. M.R.J. Kolb reports grants and personal fees from Roche, Boehringer Ingelheim and Prometic, grants from GSK, Respivert, Alkermes, Pharmaxis and Canadian Institute for Health Research, personal fees from Genoa Indalo, Third Pole and Pieris, outside the submitted work

Support statement: This work was supported by the Institute of Circulatory and Respiratory Health. Funding information for this article has been deposited with the Crossref Funder Registry.

\section{References}

1 Ley B, Collard HR, King TE. Clinical course and prediction of survival in idiopathic pulmonary fibrosis. Am J Respir Crit Care Med 2011; 183: 431-440.

2 American Thoracic Society, European Respiratory Society. American Thoracic Society/European Respiratory Society international multidisciplinary consensus classification of the idiopathic interstitial pneumonias. Am J Respir Crit Care Med 2002; 165: 277-304.

3 Hopkins RB, Burke N, Fell C, et al. Epidemiology and survival of idiopathic pulmonary fibrosis from national data in Canada. Eur Respir J 2016; 48: 187-195.

4 Nalysnyk L, Cid-Ruzafa J, Rotella P, et al. Incidence and prevalence of idiopathic pulmonary fibrosis: review of the literature. Eur Respir Rev 2012; 21: 355-361.

5 Ogura T, Taniguchi H, Azuma A, et al. Safety and pharmacokinetics of nintedanib and pirfenidone in idiopathic pulmonary fibrosis. Eur Respir J 2015; 45: 1382-1392.

6 Bonniaud P, Fabre A, Frossard N, et al. Optimising experimental research in respiratory diseases: an ERS statement. Eur Respir J 2018; 51: 1702133.

7 Landis SC, Amara SG, Asadullah K, et al. A call for transparent reporting to optimize the predictive value of preclinical research. Nature 2012; 490: 187-191. 
Tashiro J, Rubio GA, Limper AH, et al. Exploring animal models that resemble idiopathic pulmonary fibrosis. Front Med 2017; 4: 118

9 Jenkins RG, Moore BB, Chambers RC, et al. An official American Thoracic Society workshop report: use of animal models for the preclinical assessment of potential therapies for pulmonary fibrosis. Am J Respir Cell Mol Biol 2017; 56: 667-679.

10 Degryse $\mathrm{AL}$, Tanjore $\mathrm{H}, \mathrm{Xu} \mathrm{XC}$, et al. Repetitive intratracheal bleomycin models several features of idiopathic pulmonary fibrosis. Am J Physiol Lung Cell Mol Physiol 2010; 299: L442-L452.

11 Usuki J, Fukuda Y. Evolution of three patterns of intra-alveolar fibrosis produced by bleomycin in rats. Pathol Int 1995; 45: 552-564.

12 Chaudhary NI, Schnapp A, Park JE. Pharmacologic differentiation of inflammation and fibrosis in the rat bleomycin model. Am J Respir Crit Care Med 2006; 173: 769-776.

13 Kim SN, Lee J, Yang H-S, et al. Dose-response effects of bleomycin on inflammation and pulmonary fibrosis in mice. Toxicol Res 2010; 26: 217-222.

14 Izbicki G, Segel MJ, Christensen TG, et al. Time course of bleomycin-induced lung fibrosis. Int J Exp Pathol 2002; 83: 111-119.

15 Chung MP, Monick MM, Hamzeh NY, et al. Role of repeated lung injury and genetic background in bleomycin-induced fibrosis. Am J Respir Cell Mol Biol 2003; 29: 375-380.

16 Phan SH, Armstrong G, Sulavik MC, et al. A comparative study of pulmonary fibrosis induced by bleomycin and an $\mathrm{O}_{2}$ metabolite producing enzyme system. Chest 1983; 83: 44S-45S.

17 Lawson WE, Polosukhin VV, Stathopoulos GT, et al. Increased and prolonged pulmonary fibrosis in surfactant protein C-deficient mice following intratracheal bleomycin. Am J Pathol 2005; 167: 1267-1277.

18 Chua F, Gauldie J, Laurent GJ. Pulmonary fibrosis: searching for model answers. Am J Respir Cell Mol Biol 2005; 33: 9-13.

19 Limjunyawong N, Mitzner W, Horton MR. A mouse model of chronic idiopathic pulmonary fibrosis. Physiol Rep 2014; 2: e00249.

20 Peng R, Sridhar S, Tyagi G, et al. Bleomycin induces molecular changes directly relevant to idiopathic pulmonary fibrosis: a model for "active" disease. PLoS One 2013; 8: e59348.

21 Moeller A, Ask K, Warburton D, et al. The bleomycin animal model: a useful tool to investigate treatment options for idiopathic pulmonary fibrosis? Int J Biochem Cell Biol 2008; 40: 362-382.

22 Ahluwalia N, Shea BS, Tager AM. New therapeutic targets in idiopathic pulmonary fibrosis. Aiming to rein in runaway wound-healing responses. Am J Respir Crit Care Med 2014; 190: 867-878.

23 Wadman M. Animal rights: chimpanzee research on trial. Nature 2011; 474: 268-271.

24 Directive 2010/63/EU of the European Parliament and of the Council of 22 September 2010 on the Protection of Animals Used for Scientific Purposes Text with EEA Relevance. 276, 32010L0063. http://data.europa.eu/eli/dir/ 2010/63/oj/eng Date last updated: October 20, 2010.

25 Raghu G, Collard HR, Egan JJ, et al. An Official ATS/ERS/JRS/ALAT Statement: idiopathic pulmonary fibrosis: evidence-based guidelines for diagnosis and management. Am J Respir Crit Care Med 2011; 183: 788-824.

26 Noble PW, Albera C, Bradford WZ, et al. Pirfenidone in patients with idiopathic pulmonary fibrosis (CAPACITY): two randomised trials. Lancet 2011; 377: 1760-1769.

27 Margaritopoulos GA, Vasarmidi E, Antoniou KM. Pirfenidone in the treatment of idiopathic pulmonary fibrosis: an evidence-based review of its place in therapy. Core Evid 2016;11: 11-22.

28 Kakugawa T, Mukae H, Hayashi T, et al. Pirfenidone attenuates expression of HSP47 in murine bleomycin-induced pulmonary fibrosis. Eur Respir J 2004; 24: 57-65.

29 Inomata $\mathrm{M}$, Kamio $\mathrm{K}$, Azuma $\mathrm{A}$, et al. Pirfenidone inhibits fibrocyte accumulation in the lungs in bleomycin-induced murine pulmonary fibrosis. Respir Res 2014; 15 : 16.

30 Oku H, Shimizu T, Kawabata T, et al. Antifibrotic action of pirfenidone and prednisolone: different effects on pulmonary cytokines and growth factors in bleomycin-induced murine pulmonary fibrosis. Eur J Pharmacol 2008; 590: 400-408.

31 Iyer SN, Gurujeyalakshmi G, Giri SN. Effects of pirfenidone on procollagen gene expression at the transcriptional level in bleomycin hamster model of lung fibrosis. J Pharmacol Exp Ther 1999; 289: 211-218.

32 Richeldi L, du Bois RM, Raghu G, et al. Efficacy and safety of nintedanib in idiopathic pulmonary fibrosis. $N$ Engl J Med 2014; 370: 2071-2082.

33 Hajari Case A, Johnson P. Clinical use of nintedanib in patients with idiopathic pulmonary fibrosis. BMJ Open Respir Res 2017; 4: e000192.

34 Hilberg F, Roth GJ, Krssak M, et al. BIBF 1120: triple angiokinase inhibitor with sustained receptor blockade and good antitumor efficacy. Cancer Res 2008; 68: 4774-4782.

35 Hostettler KE, Zhong J, Papakonstantinou E, et al. Anti-fibrotic effects of nintedanib in lung fibroblasts derived from patients with idiopathic pulmonary fibrosis. Respir Res 2014; 15: 157.

36 The Idiopathic Pulmonary Fibrosis Clinical Research Network. A controlled trial of sildenafil in advanced idiopathic pulmonary fibrosis. N Engl J Med 2010; 363: 620-628.

37 Kolb M, Raghu G, Wells AU, et al. Nintedanib plus sildenafil in patients with idiopathic pulmonary fibrosis. N Engl J Med 2018; 379: 1722-1731.

38 King TE, Behr J, Brown KK, et al. BUILD-1: a randomized placebo-controlled trial of bosentan in idiopathic pulmonary fibrosis. Am J Respir Crit Care Med 2008; 177: 75-81.

39 King TE, Brown KK, Raghu G, et al. BUILD-3: a randomized, controlled trial of bosentan in idiopathic pulmonary fibrosis. Am J Respir Crit Care Med 2011; 184: 92-99.

40 Schroll S, Arzt M, Sebah D, et al. Improvement of bleomycin-induced pulmonary hypertension and pulmonary fibrosis by the endothelin receptor antagonist bosentan. Respir Physiol Neurobiol 2010; 170: 32-36.

41 Chilakapati SR, Serasenambati M, Vissavajjhala P, et al. Amelioration of bleomycin-induced pulmonary fibrosis in a mouse model by a combination therapy of bosentan and imatinib. Exp Lung Res 2015; 41: 173-188.

42 Zuo W-L, Zhao J-M, Huang J-X, et al. Effect of bosentan is correlated with MMP-9/TIMP-1 ratio in bleomycin-induced pulmonary fibrosis. Biomed Rep 2017; 6: 201-205.

43 Gamze K, Mehmet HM, Deveci F, et al. Effect of bosentan on the production of proinflammatory cytokines in a rat model of emphysema. Exp Mol Med 2007; 39: 614-620. 